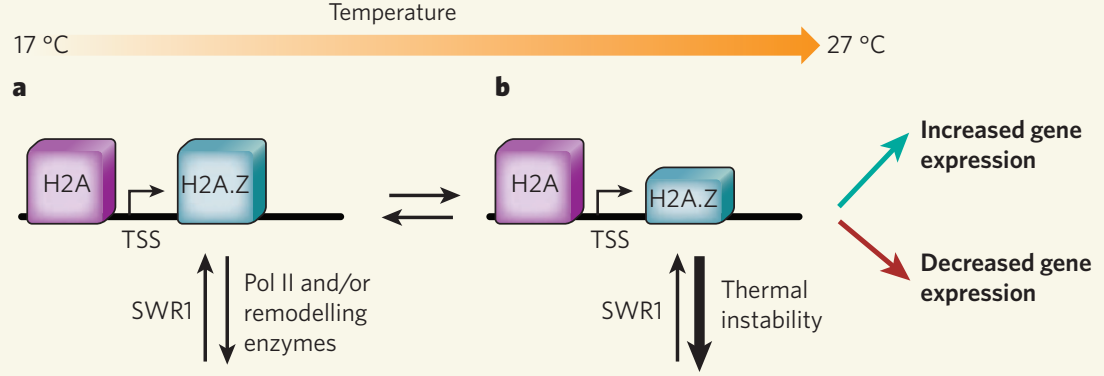

Figure 1 | Thermal regulation of gene expression by the H2A.Z protein'. a, At low temperatures, an H2A.Z-containing nucleosome is appropriately positioned just downstream of the transcriptional start site (TSS) of many genes. The abundance of this nucleosomal protein on a given gene, as indicated by the height of the H2A.Z box, reflects the balance between its deposition by the SWR1 chromatin-remodelling complex and its eviction driven by the RNA polymerase II (Pol II) enzyme or the action of chromatin-remodelling enzymes. The presence of H2A.Z-containing nucleosomes might help to maintain gene expression at a level appropriate to lower ambient temperature. $b$, As temperatures rise, the thermal instability of $\mathrm{H} 2 \mathrm{~A} . \mathrm{Z}$ pushes the equilibrium towards loss of the protein; nucleosomes containing the $\mathrm{H} 2 \mathrm{~A}$ histone protein, however, are unaffected. Depletion or modification of H2A.Z-carrying nucleosomes with rising temperature might allow gene-specific regulatory mechanisms to increase or decrease the expression of specific genes as needed for the plant to adapt to the higher temperatures.

or reduced expression of genes as needed for growth at high temperatures (Fig. 1).

Why H2A.Z has been retained as a minor variant in virtually all eukaryotes, and yet is non-essential in some, has long puzzled scientists. Examining published data, Kumar and Wigge found that changes in gene expression in yeast $\mathrm{H} 2 \mathrm{~A} . \mathrm{Z}$ mutants correlate significantly with changes that occur in response to temperature. This suggests that H2A.Z-mediated gating of the response to increased ambient temperature is an ancestral mechanism. Indeed, thermoregulation provides an attractive function that would explain the mystery of H2A.Z conservation throughout evolution, insofar as the ancestors of all organisms would have required some general mechanisms for regulating metabolism so as to respond efficiently to changes in temperature. By depositing H2A.Z over the first few nucleosomes downstream of the transcriptional start sites of essentially all genes, eukaryotes can regulate each gene independently through locally altering the amounts, modification state or dynamics of H2A.Z. Modulating the properties of H2A.Z would provide genes with a flexible gate that differentially impedes RNA polymerase II, which must disrupt nucleosomes to gain direct access to DNA and transcribe it into RNA.

There is recent evidence ${ }^{6}$ that ATP-dependent nucleosome-remodelling enzymes involved in gene regulation also recognize H2A.Zcontaining nucleosomes as being different. This finding provides additional mechanistic possibilities to explain how H2A.Z-containing nucleosomes might mediate thermoregulation as well as other processes - such as stabilization of the interaction of Polycomb-group proteins with $\mathrm{DNA}^{7}$. Kumar and Wigge's work therefore opens the door to studies of the specific molecular mechanisms underlying the responses that follow H2A.Z destabilization. It also underscores the importance of specialized nucleosomes in mediating specific transcriptional responses.

Roger B. Deal and Steven Henikoff are in the Basic Sciences Division and Howard Hughes Medical Institute, Fred Hutchinson Cancer Research Center, Seattle, Washington 98109, USA. e-mail: steveh@fhcrc.org

1. Kumar, S. V. \& Wigge, P. A. Cell 140, 136-147 (2010)

2. Choi, K. et al. Development 134, 1931-1941 (2007)

3. Deal, R. B., Topp, C. N., McKinney, E. C. \& Meagher, R. B. Plant Cell 19, 74-83 (2007)

4. Martin-Trillo, M. et al. Development 133, 1241-1252 (2006)

5. Mizuguchi, G. et al. Science 303, 343-348 (2004).

6. Goldman, J. A., Garlick, J. D. \& Kingston, R. E. J. Biol. Chem. doi:10.1074/jbc.M109.072348 (2009).

7. Creyghton, M. P. et al. Cell 135, 649-661 (2008).

\title{
nambeasuour An ill wind for finches
}

The house finch Carpodacus mexicanus, a native of North America, may have been snared by an evolutionary trap. Karen Bouwman and Dana Hawley draw this conclusion from their video evidence of feeding behaviour in experiments with caged finches (K. M. Bouwman and D. M. Hawley Biol. Lett. doi:10.1098/ rsbl.2010.0020; 2010). The trap is the preference of healthy males to feed alongside finches of the same sex that are infected with the bacterium Mycoplasma gallisepticum, so making them more vulnerable to debilitating infection by this contagious agent.

But why does this preference exist, when the expectation would be that healthy individuals would avoid their diseased fellow finches, well, like the plague? The authors' observations show that the shortterm gain for healthy males, such as the fine example pictured here, is that they have less of a battle over a meal, given that infected and so weakened males are less aggressive. Bouwman and Hawley don't rule out the involvement of chemical cues in this behaviour. But they lean towards the explanation that the visible recognition of such signs of debilitation as lethargy, and the conjunctivitis produced by M. gallisepticum infection, is the prompt.

A further curious aspect of the results is that it was males, not females, that preferred to associate with diseased individuals of the same sex at meal times. The video evidence showed that whereas healthy males were much more likely to win encounters with infected males, females showed no such edge in confrontations with infected females. There is no obvious reason why this should be so, beyond the possibility that other factors of intraspecific dominance came into play.

The bigger picture, however, concerns the evolutionary trap. House-finch infection with M. gallisepticum emerged only recently in evolutionary terms, some nine finch generations ago. Although the bacterium has since produced seasonal epidemics in finch populations, Bouwman and Hawley's view is that the birds have not yet caught on to the full significance of the signs

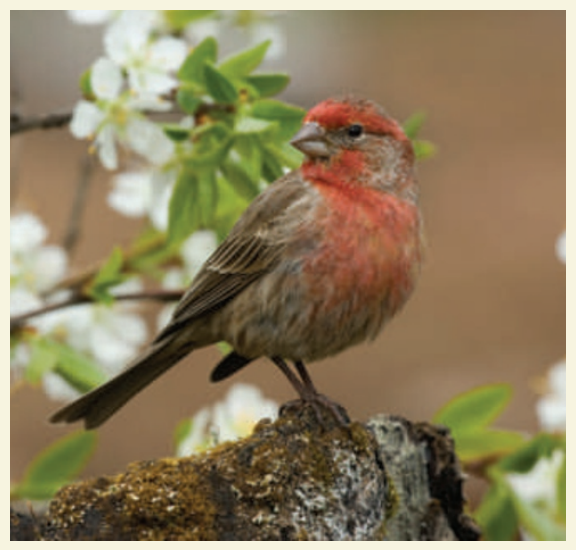

of disease. Rather, the males, at least, continue to interpret these 'sickness behaviours' not as warnings to keep clear, but as indications that they have simply picked a loser in contests for food. Such a misapprehension, say the authors, can only contribute to the persistence of disease epidemics among these birds. Tim Lincoln 